\title{
Ring Fusion Effects on the Solid-State Properties of $\alpha$-Oligothiophenes
}

\author{
Xinnan Zhang, James P. Johnson, Jeff W. Kampf, and Adam J. Matzger* \\ Department of Chemistry and the Macromolecular Science and Engineering Program, \\ University of Michigan, 930 N. University, Ann Arbor, MI 48109-1055 \\ matzger@umich.edu
}

Table of contents

1. Crystallographic Information and structure refinement for compounds 1-4

2. Powder X-ray diffraction (PXRD) data.

3. Energy profile calculation of compounds $\mathbf{3}$ and $\mathbf{4}$

4. Electronic transition calculations of compound $\mathbf{2}$ (planar conformation vs. curved conformation).

5. All calculated electronic transitions

1. Crystallographic Information and structure refinement for compounds 1-4

Table 1. Crystallographic Information and structure refinement for compound $\mathbf{1 .}$

$\begin{array}{ll}\text { Identification code } & \mathrm{tt} 2 \mathrm{t} \\ \text { Empirical formula } & \mathrm{C} 14 \mathrm{H} 8 \mathrm{~S} 4 \\ \text { Formula weight } & 304.44 \\ \text { Temperature } & 123(2) \mathrm{K} \\ \text { Wavelength } & 0.71073 \mathrm{~A} \\ \text { Crystal system, space group } & \text { Triclinic, P-1 } \\ \text { Unit cell dimensions } & \mathrm{a}=5.7083(15) \mathrm{A} \text { alpha }=88.226(6) \mathrm{deg} . \\ & \mathrm{b}=8.616(2) \mathrm{A} \text { beta }=77.637(6) \mathrm{deg} . \\ & \mathrm{c}=13.272(4) \mathrm{A} \text { gamma }=82.068(6) \mathrm{deg} . \\ \text { Volume } & 631.5(3) \mathrm{A} \wedge 3 \\ \text { Z, Calculated density } & 2,1.601 \mathrm{Mg} / \mathrm{m}^{\wedge} 3 \\ \text { Absorption coefficient } & 0.726 \mathrm{~mm}^{\wedge}-1 \\ \text { F(000) } & 312\end{array}$


Crystal size

Theta range for data collection

Limiting indices

Reflections collected / unique

Completeness to theta $=26.51$

Absorption correction

Max. and min. transmission

Refinement method

Data / restraints / parameters

Goodness-of-fit on $\mathrm{F}^{\wedge} 2$

Final $\mathrm{R}$ indices [I $>2 \operatorname{sigma}(\mathrm{I})]$

$\mathrm{R}$ indices (all data)

Largest diff. peak and hole
$0.30 \times 0.20 \times 0.02 \mathrm{~mm}$

2.86 to $26.51 \mathrm{deg}$.

$-7<=\mathrm{h}<=7,-10<=\mathrm{k}<=10,-16<=\mathrm{l}<=16$

$9522 / 2602[\mathrm{R}($ int $)=0.0613]$

$99.2 \%$

Semi-empirical from equivalents

0.9856 and 0.8115

Full-matrix least-squares on $\mathrm{F}^{\wedge} 2$

$2602 / 0 / 168$

1.025

$\mathrm{R} 1=0.0567, \mathrm{wR} 2=0.1436$

$\mathrm{R} 1=0.0773, \mathrm{wR} 2=0.1541$

0.508 and -0.624 e. $\mathrm{A}^{\wedge}-3$

Table 2. Crystallographic Information and structure refinement for compound 2.

$\begin{array}{ll}\text { Identification code } & \mathrm{t} 2 \mathrm{tt} \\ \text { Empirical formula } & \mathrm{C} 14 \mathrm{H} 8 \mathrm{~S} 4 \\ \text { Formula weight } & 304.44 \\ \text { Temperature } & 123(2) \mathrm{K} \\ \text { Wavelength } & 0.71073 \mathrm{~A} \\ \text { Crystal system, space group } & \text { Monoclinic, P2(1) } \\ \text { Unit cell dimensions } & \begin{array}{l}\mathrm{a}=7.4265(11) \mathrm{A} \text { alpha }=90 \mathrm{deg} . \\ \mathrm{b}=5.7172(8) \mathrm{A} \text { beta }=102.336(2) \mathrm{deg} . \\ \mathrm{c}=14.840(2) \mathrm{A} \text { gamma }=90 \mathrm{deg} .\end{array}\end{array}$


Volume

Z, Calculated density

Absorption coefficient

$\mathrm{F}(000)$

Crystal size

Theta range for data collection

Limiting indices

Reflections collected / unique

Completeness to theta $=20.77$

Absorption correction

Max. and min. transmission

Refinement method

Data / restraints / parameters

Goodness-of-fit on $\mathrm{F}^{\wedge} 2$

Final $\mathrm{R}$ indices [I $>2 \operatorname{sigma}(\mathrm{I})]$

$\mathrm{R}$ indices (all data)

Absolute structure parameter

Largest diff. peak and hole
$615.53(15) \mathrm{A}^{\wedge} 3$

2, $1.643 \mathrm{Mg} / \mathrm{m}^{\wedge} 3$

$0.745 \mathrm{~mm}^{\wedge}-1$

312

$0.20 \times 0.16 \times 0.04 \mathrm{~mm}$

2.81 to $20.77 \mathrm{deg}$.

$-7<=\mathrm{h}<=7,-5<=\mathrm{k}<=5,-14<=\mathrm{l}<=14$

$7063 / 1286[\mathrm{R}($ int $)=0.0959]$

$99.9 \%$

Semi-empirical from equivalents

0.9852 and 0.8652

Full-matrix least-squares on $\mathrm{F}^{\wedge} 2$

$1286 / 1 / 165$

0.930

$\mathrm{R} 1=0.0577, \mathrm{wR} 2=0.1468$

$\mathrm{R} 1=0.0937, \mathrm{wR} 2=0.1700$

$0.4(4)$

0.543 and -0.446 e. $\mathrm{A}^{\wedge}-3$

Table 3. Crystallographic Information and structure refinement for compound $\mathbf{3}$.

Identification code

Empirical formula $\mathrm{t} 2$

C12 H6 S4 
Formula weight

Temperature

Wavelength

Crystal system, space group

Unit cell dimensions

Volume

Z, Calculated density

Absorption coefficient

$\mathrm{F}(000)$

Crystal size

Theta range for data collection

Limiting indices

Reflections collected / unique

Completeness to theta $=31.67$

Absorption correction

Max. and min. transmission

Refinement method

Data / restraints / parameters

Goodness-of-fit on $\mathrm{F}^{\wedge} 2$

Final $\mathrm{R}$ indices [I $>2 \operatorname{sigma}(\mathrm{I})]$

$\mathrm{R}$ indices (all data)

Largest diff. peak and hole
278.41

153(2) K

$0.77500 \mathrm{~A}$

Triclinic, $\mathrm{P}-1$

$\mathrm{a}=5.7668(16)$ A alpha $=85.131(7) \mathrm{deg}$.

$\mathrm{b}=7.551(2) \mathrm{A} \quad$ beta $=88.367(7) \mathrm{deg}$.

$\mathrm{c}=12.884(4) \mathrm{A}$ gamma $=89.927(8) \mathrm{deg}$.

$558.8(3) \mathrm{A}^{\wedge} 3$

2, $1.655 \mathrm{Mg} / \mathrm{m}^{\wedge} 3$

$0.812 \mathrm{~mm}^{\wedge}-1$

284

$0.20 \times 0.02 \times 0.02 \mathrm{~mm}$

1.73 to $31.67 \mathrm{deg}$.

$-7<=\mathrm{h}<=7,-9<=\mathrm{k}<=10,-17<=\mathrm{l}<=17$

$6121 / 2750[\mathrm{R}($ int $)=0.0603]$

$94.8 \%$

Semi-empirical from equivalents

0.9839 and 0.8544

Full-matrix least-squares on $\mathrm{F}^{\wedge} 2$

$2750 / 0 / 146$

1.125

$\mathrm{R} 1=0.0810, \mathrm{wR} 2=0.2328$

$\mathrm{R} 1=0.0972, \mathrm{wR} 2=0.2434$

1.172 and -1.304 e. $\mathrm{A}^{\wedge}-3$ 
Table 4. Crystallographic Information and structure refinement for compound 4.

Identification code $\quad \mathrm{t} 3 \mathrm{t}$

Empirical formula $\quad$ C12 H6 S4

Formula weight $\quad 278.41$

Temperature $\quad$ 123(2) K

Wavelength $\quad 0.71073 \mathrm{~A}$

Crystal system, space group Orthorhombic, Pca2(1)

Unit cell dimensions $\quad \mathrm{a}=23.660(5) \mathrm{A}$ alpha $=90 \mathrm{deg}$.

$\mathrm{b}=5.9832(12) \mathrm{A} \quad$ beta $=90 \mathrm{deg}$.

$\mathrm{c}=15.709(3)$ A gamma $=90 \mathrm{deg}$.

Volume

2223.7(8) $\mathrm{A}^{\wedge} 3$

Z, Calculated density

$8,1.663 \mathrm{Mg} / \mathrm{m}^{\wedge} 3$

Absorption coefficient

$0.817 \mathrm{~mm}^{\wedge}-1$

$\mathrm{F}(000)$

1136

Crystal size

$0.40 \times 0.04 \times 0.02 \mathrm{~mm}$

Theta range for data collection

3.11 to $28.31 \mathrm{deg}$.

Limiting indices

$-31<=\mathrm{h}<=31,-7<=\mathrm{k}<=7,-20<=\mathrm{l}<=20$

Reflections collected / unique

$24536 / 2848[\mathrm{R}($ int $)=0.0832]$

Completeness to theta $=28.31 \quad 99.7 \%$

Absorption correction Semi-empirical from equivalents

Max. and min. transmission $\quad 0.9839$ and 0.7359 
Refinement method

Data / restraints / parameters

Goodness-of-fit on $\mathrm{F}^{\wedge} 2$

Final $R$ indices [I $>2 \operatorname{sigma}(\mathrm{I})]$

$\mathrm{R}$ indices (all data)

Absolute structure parameter

Largest diff. peak and hole
Full-matrix least-squares on $\mathrm{F}^{\wedge} 2$

$2848 / 1 / 289$

1.012

$\mathrm{R} 1=0.0341, \mathrm{wR} 2=0.0755$

$\mathrm{R} 1=0.0596, \mathrm{wR} 2=0.0839$

$0.08(13)$

0.491 and -0.353 e. $\mathrm{A}^{\wedge}-3$

2. Powder X-ray diffraction (PXRD) data

PXRD data were recorded at room temperature on a Bruker AXS $\mathrm{D}_{8}$ Advance powder diffractometer with a CuK $\alpha$ source $(40 \mathrm{kV}, 40 \mathrm{~mA}, \lambda=1.5406 \AA)$ with a scan speed of $0.01 \mathrm{sec} / \mathrm{step}$ and a step size of $0.01^{\circ}$. The samples used for PXRD experiments were also employed for solid-state UV-visible measurements. PXRD of films gave very few peaks due to the preferred orientation and the small sample size that resulted in weak diffraction. It has been confirmed that all peaks presented in the powder X-ray spectra exist in the simulated PXRD patterns calculated from the crystal structures.

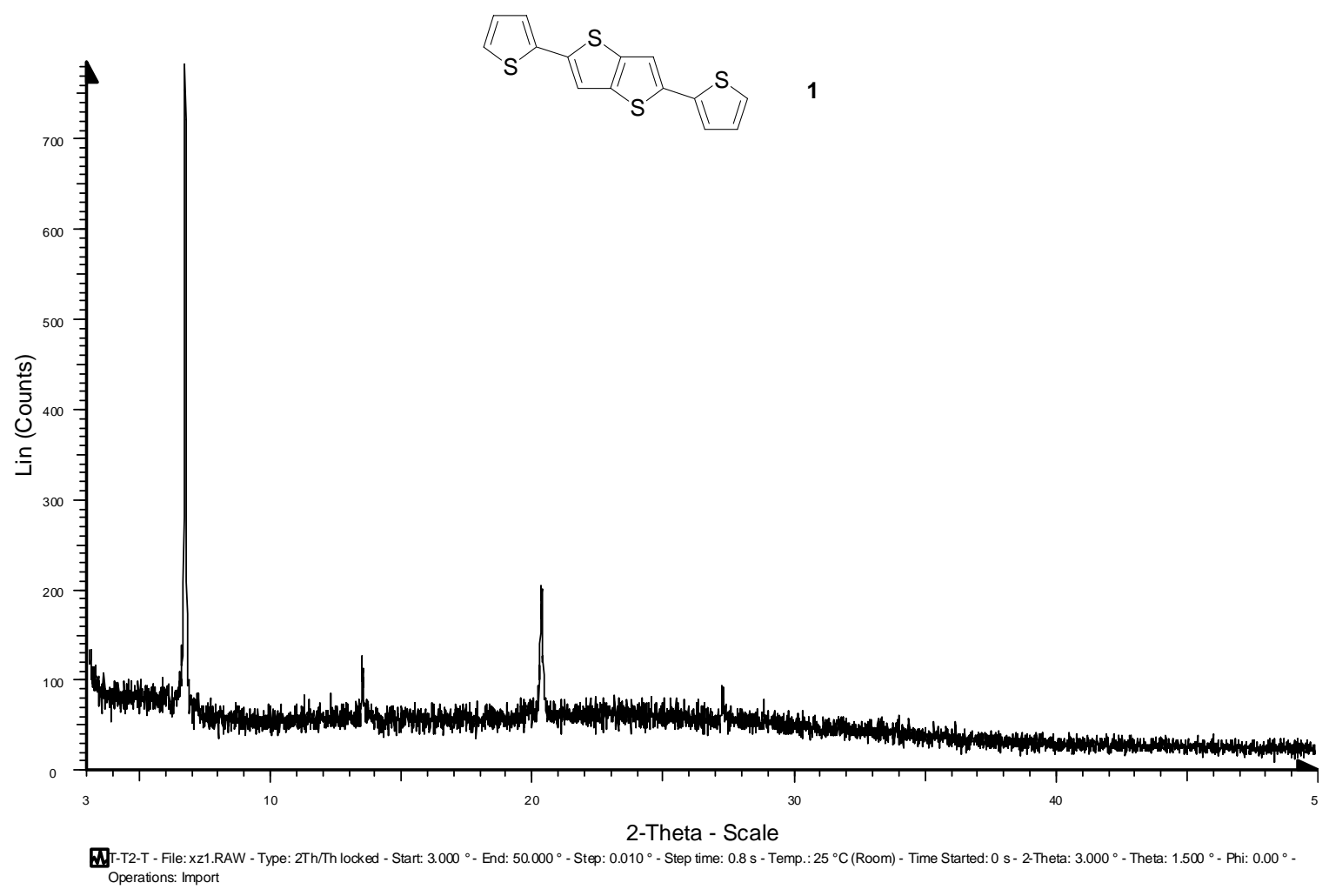




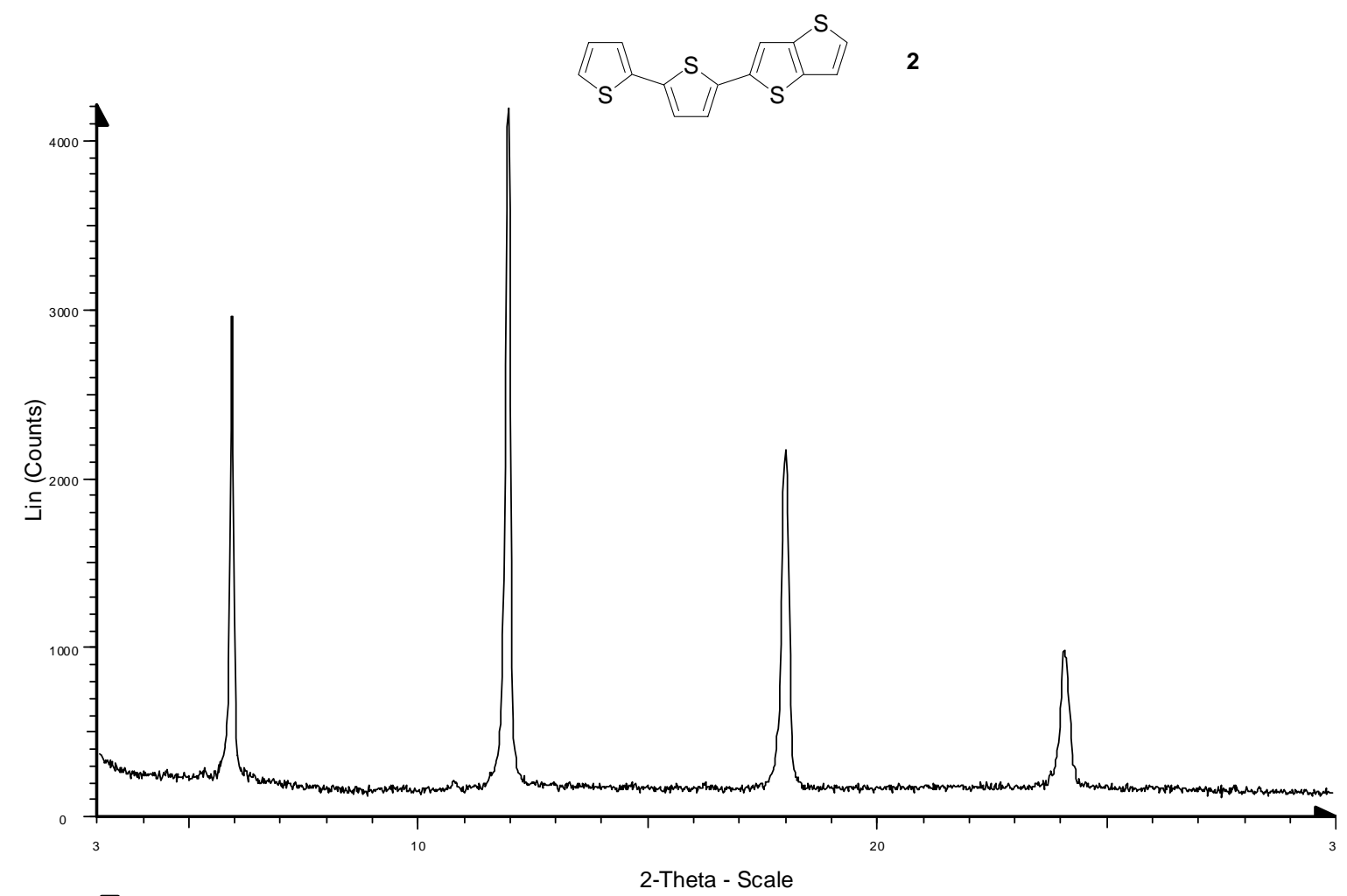

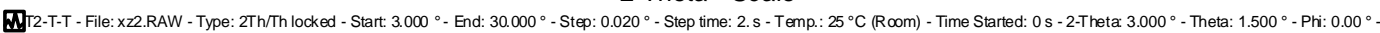
Operations: Import

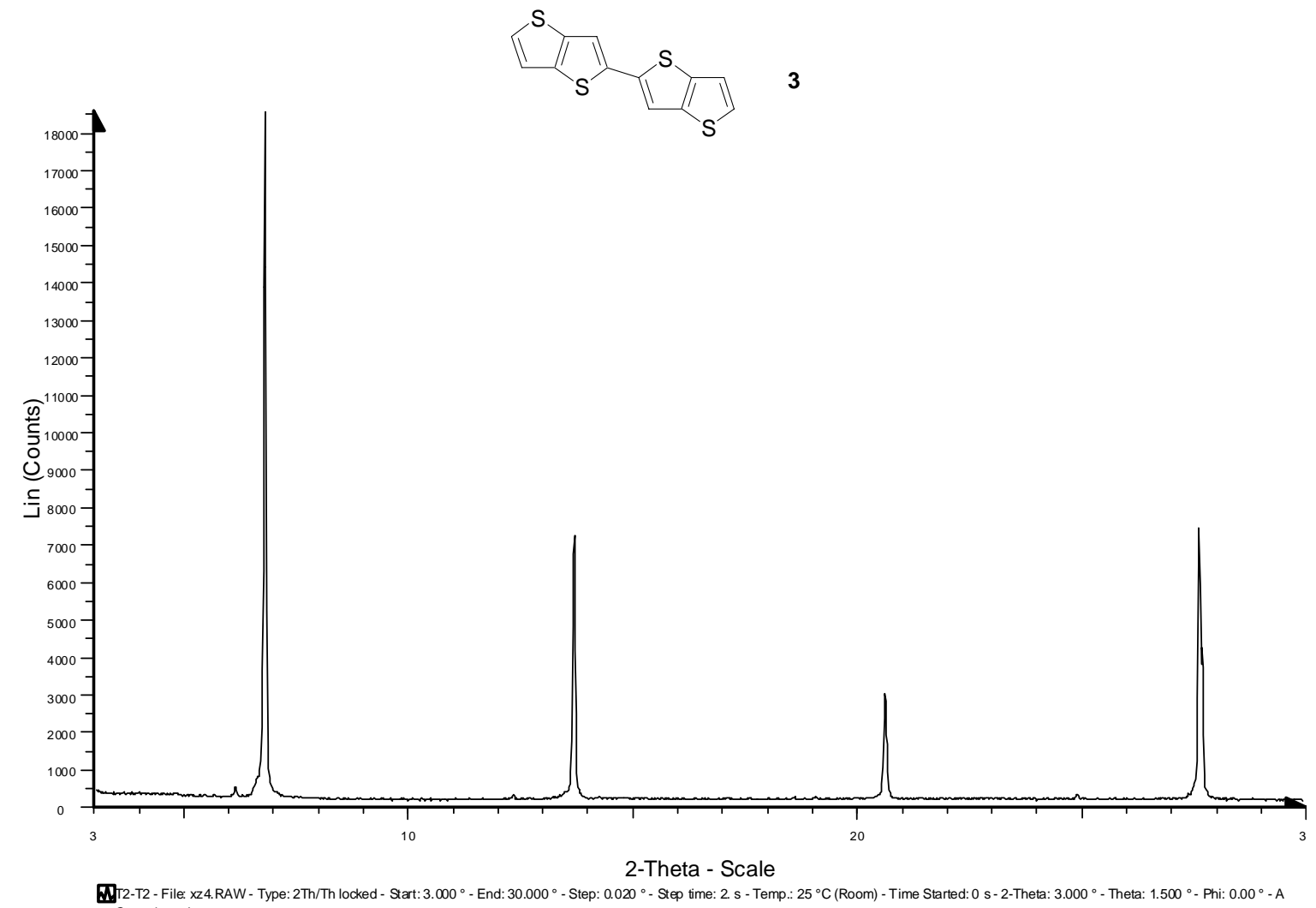

WT2-T2 - File: xz4.RAW - Type: 2 Th/Th locked - Start: $3.000^{\circ}$ - End: $30.000^{\circ}$ - Step: $0.020^{\circ}$ - Step time: $2 \mathrm{~s}$ - Temp.: $25^{\circ} \mathrm{C}$ (Room) - Time Started: 0 s - 2-Theta: $3.000^{\circ}$ - Theta: $1.500^{\circ}$ - Phi: $0.00^{\circ}$ - A Operations: Import 

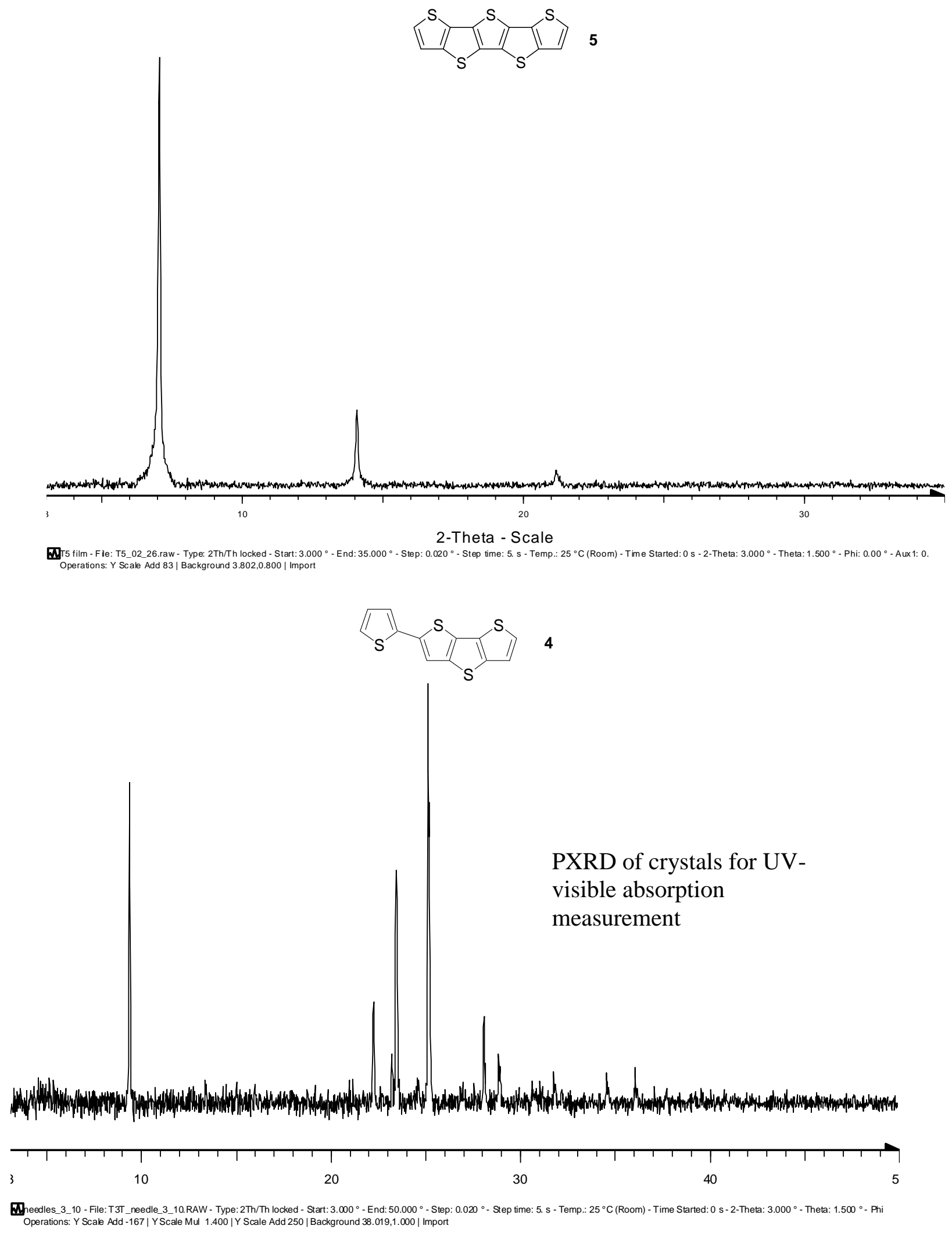
3. Energy profile calculations of compounds 3 and $\mathbf{4}$.

The energy profiles of $\mathbf{3}$ and $\mathbf{4}$ were examined with density functional theory at the B3LYP/6-31G* level. In each case the energy was computed for 31 different torsion angles (defined between the carbon atoms linking the two thiophene rings) from 0 to $90^{\circ}$.
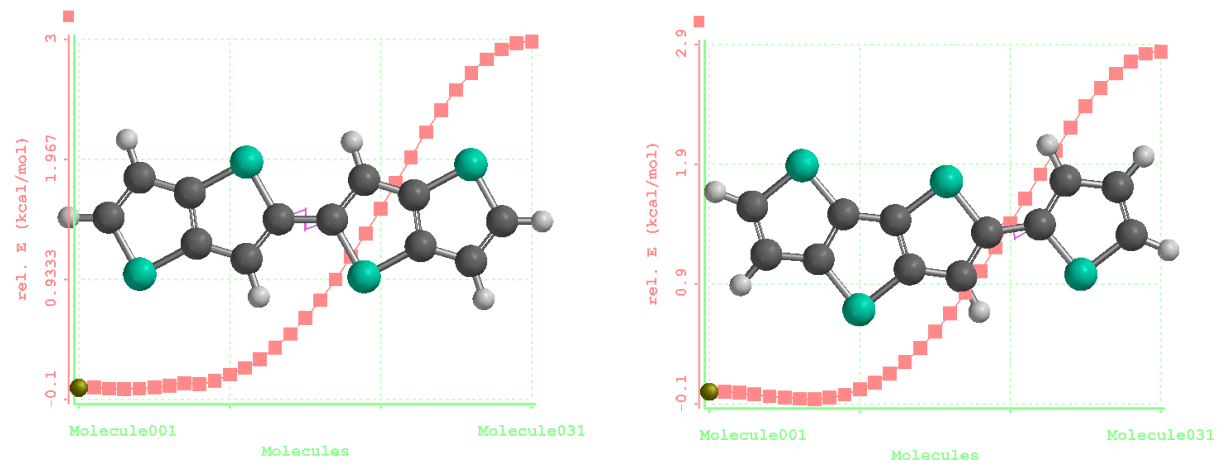

4. Electronic transition calculations of compound $\mathbf{2}$ (planar conformation vs. curved conformation).

Table 5. Calculated transitions of the curved and planar conformations of compound 2.

\begin{tabular}{|c|c|}
\hline conformation of compound 2 & $\begin{array}{c}\text { calculated } \lambda_{\max } \text { in nanometers } \\
\text { (intensity) }\end{array}$ \\
\hline -caneseco-cenes-cosed $=$ & $389.4(0.932)$ \\
\hline c-cacenseo-caces-oreco-o & $389.3(0.996)$ \\
\hline
\end{tabular}


5. All calculated electronic transitions

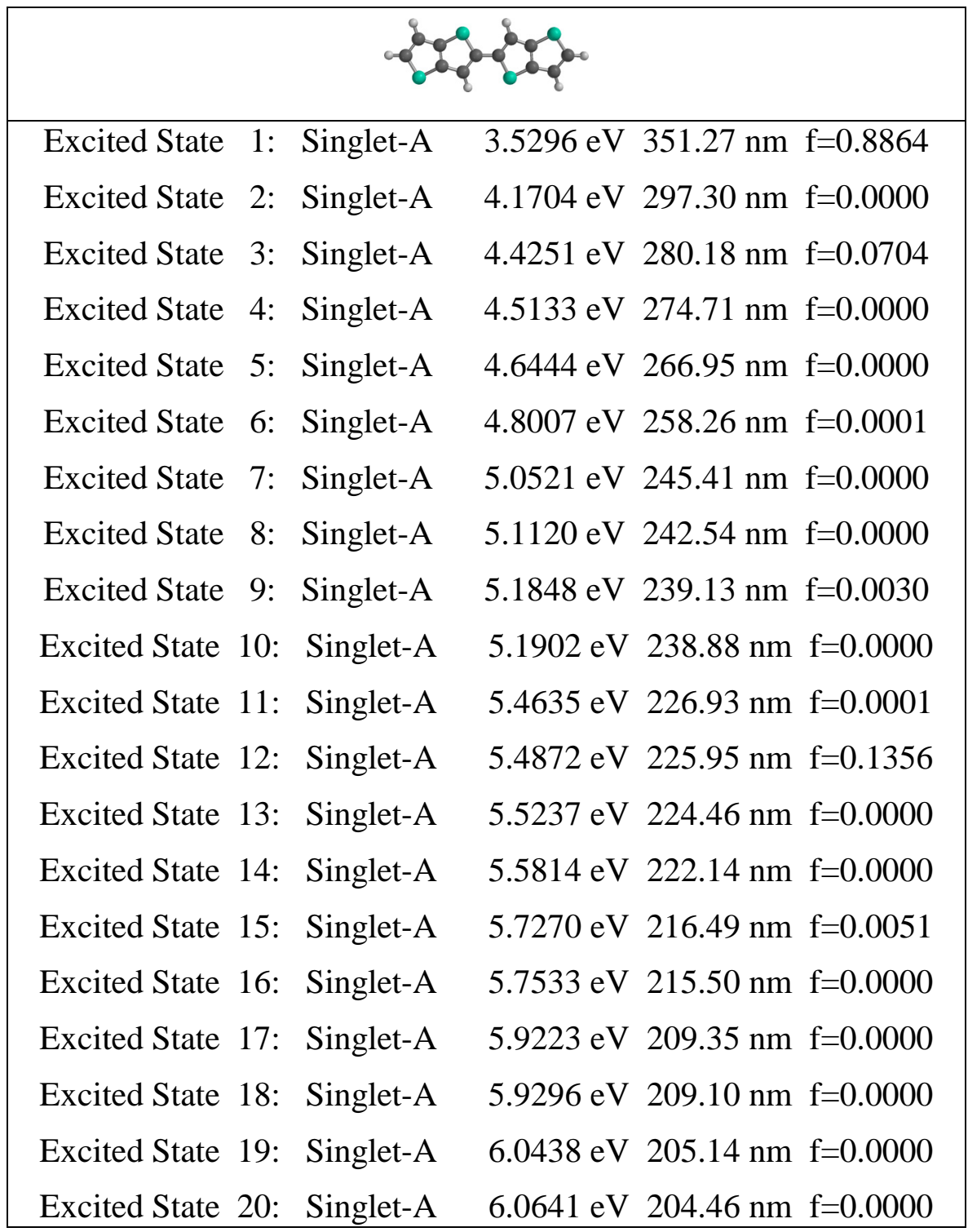

\begin{tabular}{|c|c|c|}
\hline \multicolumn{3}{|c|}{ 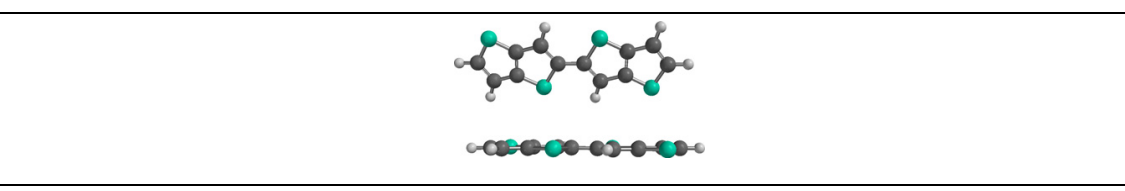 } \\
\hline Excited State & 1: Singlet-A & $3.0472 \mathrm{eV} 406.88 \mathrm{~nm} \mathrm{f}=0.0029$ \\
\hline Excited State & 2: Singlet-A & $3.4814 \mathrm{eV} \quad 356.13 \mathrm{~nm} \mathrm{f}=0.0086$ \\
\hline Excited State & 3: Singlet-A & $3.6351 \mathrm{eV} 341.07 \mathrm{~nm} \mathrm{f}=0.1626$ \\
\hline Excited State & 4: Singlet-A & $3.7266 \mathrm{eV} \quad 332.70 \mathrm{~nm} \mathrm{f}=1.3121$ \\
\hline Excited State & 5: Singlet-A & $3.8378 \mathrm{eV} \quad 323.06 \mathrm{~nm} f=0.0177$ \\
\hline Excited State & 6: Singlet-A & $4.0453 \mathrm{eV} \quad 306.49 \mathrm{~nm} \mathrm{f}=0.0322$ \\
\hline
\end{tabular}




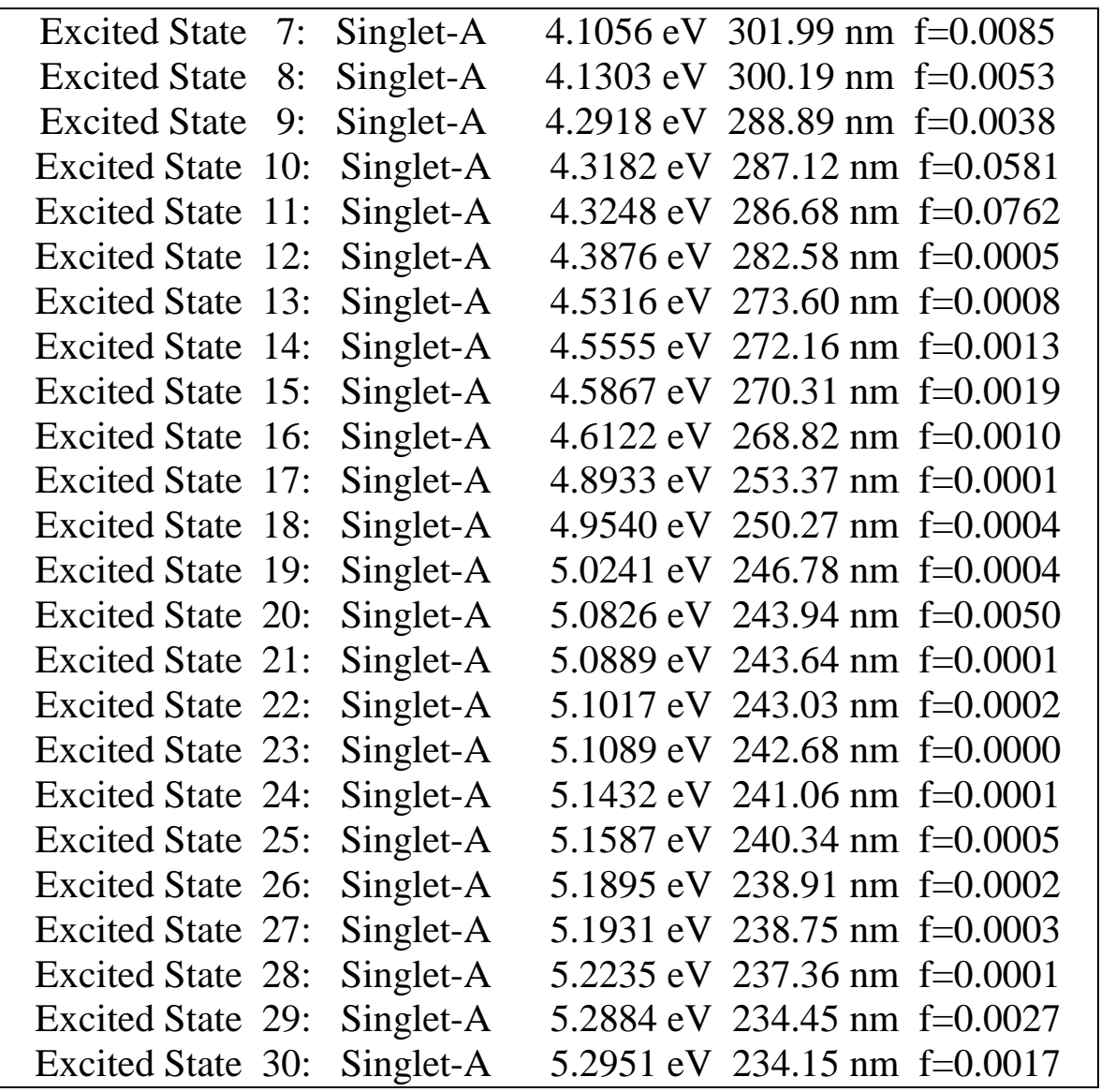

\begin{tabular}{|c|c|c|}
\hline Excited State & Singlet-A & $3.5153 \mathrm{eV} 352.70 \mathrm{~nm} \mathrm{f}=0.7784$ \\
\hline Excited State & Singlet-A & $4.0781 \mathrm{eV} 304.02 \mathrm{~nm} \mathrm{f}=0.0508$ \\
\hline Excited State & Singlet-A & $4.4279 \mathrm{eV} 280.00 \mathrm{~nm} \mathrm{f}=0.0096$ \\
\hline Excited State & Singlet-A & $4.5190 \mathrm{eV} 274.36 \mathrm{~nm} \mathrm{f}=0.0002$ \\
\hline Excited State & Singlet-A & $4.7540 \mathrm{eV} 260.80 \mathrm{~nm} \mathrm{f}=0.0000$ \\
\hline Excited State & Singlet-A & $4.9026 \mathrm{eV} 252.89 \mathrm{~nm} \mathrm{f}=0.0180$ \\
\hline Excited State & Singlet-A & $4.9410 \mathrm{eV} 250.93 \mathrm{~nm} \mathrm{f}=0.0138$ \\
\hline Excited State & Singlet-A & $5.0898 \mathrm{eV} 243.59 \mathrm{~nm} \mathrm{f}=0.0006$ \\
\hline Excited State 9 & Singlet-A & $5.1713 \mathrm{eV} 239.76 \mathrm{~nm} \mathrm{f}=0.0216$ \\
\hline Excited State 10 & Singlet-A & $5.2122 \mathrm{eV} \quad 237.87 \mathrm{~nm} \mathrm{f}=0.0186$ \\
\hline Excited State 11 & Singlet-A & $5.2746 \mathrm{eV} \quad 235.06 \mathrm{~nm} \mathrm{f}=0.0202$ \\
\hline Excited State 12 & Singlet-A & $5.3018 \mathrm{eV} \quad 233.86 \mathrm{~nm} \mathrm{f}=0.0062$ \\
\hline Excited State 13 & Singlet-A & $5.4748 \mathrm{eV} 226.46 \mathrm{~nm} \mathrm{f}=0.0012$ \\
\hline Excited State 14 & Singlet-A & $5.6329 \mathrm{eV} 220.11 \mathrm{~nm} \mathrm{f}=0.0003$ \\
\hline Excited State 15 & Singlet-A & $5.8963 \mathrm{eV} \quad 210.27 \mathrm{~nm} \mathrm{f}=0.0103$ \\
\hline Excited State 16 & Singlet-A & $5.9358 \mathrm{eV} 208.88 \mathrm{~nm} \mathrm{f}=0.0001$ \\
\hline Excited State 17 & Singlet-A & $6.0359 \mathrm{eV} 205.41 \mathrm{~nm} \mathrm{f}=0.0085$ \\
\hline Excited State 18 & Singlet-A & $6.0646 \mathrm{eV} 204.44 \mathrm{~nm} \mathrm{f}=0.0024$ \\
\hline
\end{tabular}


Excited State 19: Singlet-A $\quad 6.1144 \mathrm{eV} 202.77 \mathrm{~nm} \mathrm{f}=0.0231$

Excited State 20: Singlet-A $\quad 6.1414 \mathrm{eV} \quad 201.88 \mathrm{~nm} \mathrm{f}=0.0115$

\begin{tabular}{|lllll|}
\hline & & \\
& &
\end{tabular}

\begin{tabular}{|lllll|}
\hline & \multicolumn{4}{|c|}{} \\
\hline Excited State & 1: & Singlet-A & $3.1535 \mathrm{eV}$ & $393.16 \mathrm{~nm} \mathrm{f}=0.0973$ \\
Excited State & 2: & Singlet-A & $3.3212 \mathrm{eV}$ & $373.31 \mathrm{~nm} \mathrm{f}=0.1974$ \\
Excited State & 3: & Singlet-A & $3.4393 \mathrm{eV}$ & $360.49 \mathrm{~nm} \mathrm{f}=0.1431$ \\
\hline
\end{tabular}




\begin{tabular}{|c|c|c|c|}
\hline Excited State & 4: & Singlet-A & $3.5382 \mathrm{eV} 350.42 \mathrm{~nm} \mathrm{f}=1.0808$ \\
\hline Excited State & 5: & Singlet-A & $3.8586 \mathrm{eV} 321.32 \mathrm{~nm} \mathrm{f}=0.0189$ \\
\hline Excited State & 6: & Singlet-A & $3.9870 \mathrm{eV} 310.97 \mathrm{~nm} \mathrm{f}=0.0197$ \\
\hline Excited State & 7: & Singlet-A & $4.1314 \mathrm{eV} 300.10 \mathrm{~nm} \mathrm{f}=0.0348$ \\
\hline Excited State & 8: & Singlet-A & $4.2873 \mathrm{eV} 289.19 \mathrm{~nm} \mathrm{f}=0.0070$ \\
\hline Excited State & 9: & Singlet-A & $4.3559 \mathrm{eV} 284.63 \mathrm{~nm} \mathrm{f}=0.0146$ \\
\hline Excited State & 10: & Singlet-A & $4.4064 \mathrm{eV} 281.37 \mathrm{~nm} \mathrm{f}=0.0064$ \\
\hline Excited State & 11: & Singlet-A & $4.4416 \mathrm{eV} 279.14 \mathrm{~nm} \mathrm{f}=0.0009$ \\
\hline Excited State & 12: & Singlet-A & $4.4573 \mathrm{eV} 278.16 \mathrm{~nm} \mathrm{f}=0.0021$ \\
\hline Excited State & 13: & Singlet-A & $4.4776 \mathrm{eV} 276.90 \mathrm{~nm} \mathrm{f}=0.0015$ \\
\hline Excited State & 14: & Singlet-A & $4.5609 \mathrm{eV} 271.84 \mathrm{~nm} \mathrm{f}=0.0016$ \\
\hline Excited State & 15: & Singlet-A & $4.6359 \mathrm{eV} 267.44 \mathrm{~nm} \mathrm{f}=0.0012$ \\
\hline Excited State & 16: & Singlet-A & $4.7010 \mathrm{eV} 263.74 \mathrm{~nm} \mathrm{f}=0.0005$ \\
\hline Excited State & 17: & Singlet-A & $4.7319 \mathrm{eV} 262.02 \mathrm{~nm} \mathrm{f}=0.0003$ \\
\hline Excited State & 18: & Singlet-A & $4.7407 \mathrm{eV} 261.53 \mathrm{~nm} \mathrm{f}=0.0006$ \\
\hline Excited State & 19: & Singlet-A & $4.8315 \mathrm{eV} 256.62 \mathrm{~nm} \mathrm{f}=0.0184$ \\
\hline Excited State & 20: & Singlet-A & $4.8470 \mathrm{eV} 255.80 \mathrm{~nm} \mathrm{f}=0.0005$ \\
\hline Excited State & 21: & Singlet-A & $4.8765 \mathrm{eV} 254.25 \mathrm{~nm} \mathrm{f}=0.0078$ \\
\hline Excited State & 22: & Singlet-A & $4.9184 \mathrm{eV} 252.08 \mathrm{~nm} \mathrm{f}=0.0156$ \\
\hline Excited State & 23: & Singlet-A & $4.9388 \mathrm{eV} 251.04 \mathrm{~nm} \mathrm{f}=0.0006$ \\
\hline Excited State & 24: & Singlet-A & $4.9459 \mathrm{eV} 250.68 \mathrm{~nm} \mathrm{f}=0.0044$ \\
\hline Excited State & 25: & Singlet-A & $4.9707 \mathrm{eV} 249.43 \mathrm{~nm} \mathrm{f}=0.0014$ \\
\hline Excited State & 26: & Singlet-A & $4.9864 \mathrm{eV} 248.64 \mathrm{~nm} \mathrm{f}=0.0081$ \\
\hline Excited State & 27: & Singlet-A & $4.9894 \mathrm{eV} 248.50 \mathrm{~nm} \mathrm{f}=0.0003$ \\
\hline Excited State & 28: & Singlet-A & $5.0571 \mathrm{eV} 245.17 \mathrm{~nm} \mathrm{f}=0.0012$ \\
\hline Excited State & 29: & Singlet-A & $5.1203 \mathrm{eV} 242.14 \mathrm{~nm} \mathrm{f}=0.0144$ \\
\hline Excited State & 30: & Singlet-A & $5.1272 \mathrm{eV} 241.81 \mathrm{~nm} \mathrm{f}=0.0090$ \\
\hline
\end{tabular}

\begin{tabular}{|c|c|c|}
\hline Excited State & 1: Singlet-A & $3.6257 \mathrm{eV} 341.96 \mathrm{~nm} \mathrm{f}=0.7565$ \\
\hline Excited State & 2: Singlet-A & $4.0798 \mathrm{eV} 303.90 \mathrm{~nm} \mathrm{f}=0.1061$ \\
\hline Excited State & 3: Singlet-A & $4.3167 \mathrm{eV} 287.22 \mathrm{~nm} \mathrm{f}=0.0001$ \\
\hline Excited State & 4: Singlet-A & $4.3312 \mathrm{eV} 286.26 \mathrm{~nm} \mathrm{f}=0.0000$ \\
\hline Excited State & 5: Singlet-A & $4.5562 \mathrm{eV} 272.12 \mathrm{~nm} \mathrm{f}=0.0004$ \\
\hline Excited State & 6: Singlet-A & $4.5973 \mathrm{eV} 269.69 \mathrm{~nm} \mathrm{f}=0.0041$ \\
\hline Excited State & 7: Singlet-A & $4.8879 \mathrm{eV} 253.66 \mathrm{~nm} \mathrm{f}=0.0000$ \\
\hline Excited State & 8: Singlet-A & $4.9257 \mathrm{eV} 251.71 \mathrm{~nm} \mathrm{f}=0.0000$ \\
\hline Excited State & 9: Singlet-A & $5.0477 \mathrm{eV} 245.63 \mathrm{~nm} \mathrm{f}=0.0000$ \\
\hline Excited State & 10: Singlet-A & $5.1316 \mathrm{eV} 241.61 \mathrm{~nm} \mathrm{f}=0.0127$ \\
\hline Excited State & 11: Singlet-A & $5.1642 \mathrm{eV} 240.08 \mathrm{~nm} \mathrm{f}=0.0000$ \\
\hline Excited State & 12: Singlet-A & $5.2272 \mathrm{eV} 237.19 \mathrm{~nm} \mathrm{f}=0.0012$ \\
\hline Excited State & 13: Singlet-A & $5.2772 \mathrm{eV} \quad 234.95 \mathrm{~nm} \mathrm{f}=0.0013$ \\
\hline Excited State & 14: Singlet-A & $5.3341 \mathrm{eV} \quad 232.44 \mathrm{~nm} \mathrm{f}=0.0001$ \\
\hline Excited State & 15: Singlet-A & $5.3812 \mathrm{eV} 230.40 \mathrm{~nm} \mathrm{f}=0.0001$ \\
\hline
\end{tabular}




\begin{tabular}{|lllll|}
\hline Excited State & $16:$ & Singlet-A & $5.4464 \mathrm{eV}$ & $227.64 \mathrm{~nm} \mathrm{f}=0.0014$ \\
Excited State 17: & Singlet-A & $5.5319 \mathrm{eV}$ & $224.12 \mathrm{~nm} \mathrm{f}=0.0000$ \\
Excited State 18: & Singlet-A & $5.7908 \mathrm{eV}$ & $214.10 \mathrm{~nm} \mathrm{f}=0.0534$ \\
Excited State 19: & Singlet-A & $5.8414 \mathrm{eV}$ & $212.25 \mathrm{~nm} \mathrm{f}=0.0000$ \\
Excited State & 20: & Singlet-A & $5.9331 \mathrm{eV}$ & $208.97 \mathrm{~nm} \mathrm{f}=0.0002$ \\
\hline
\end{tabular}

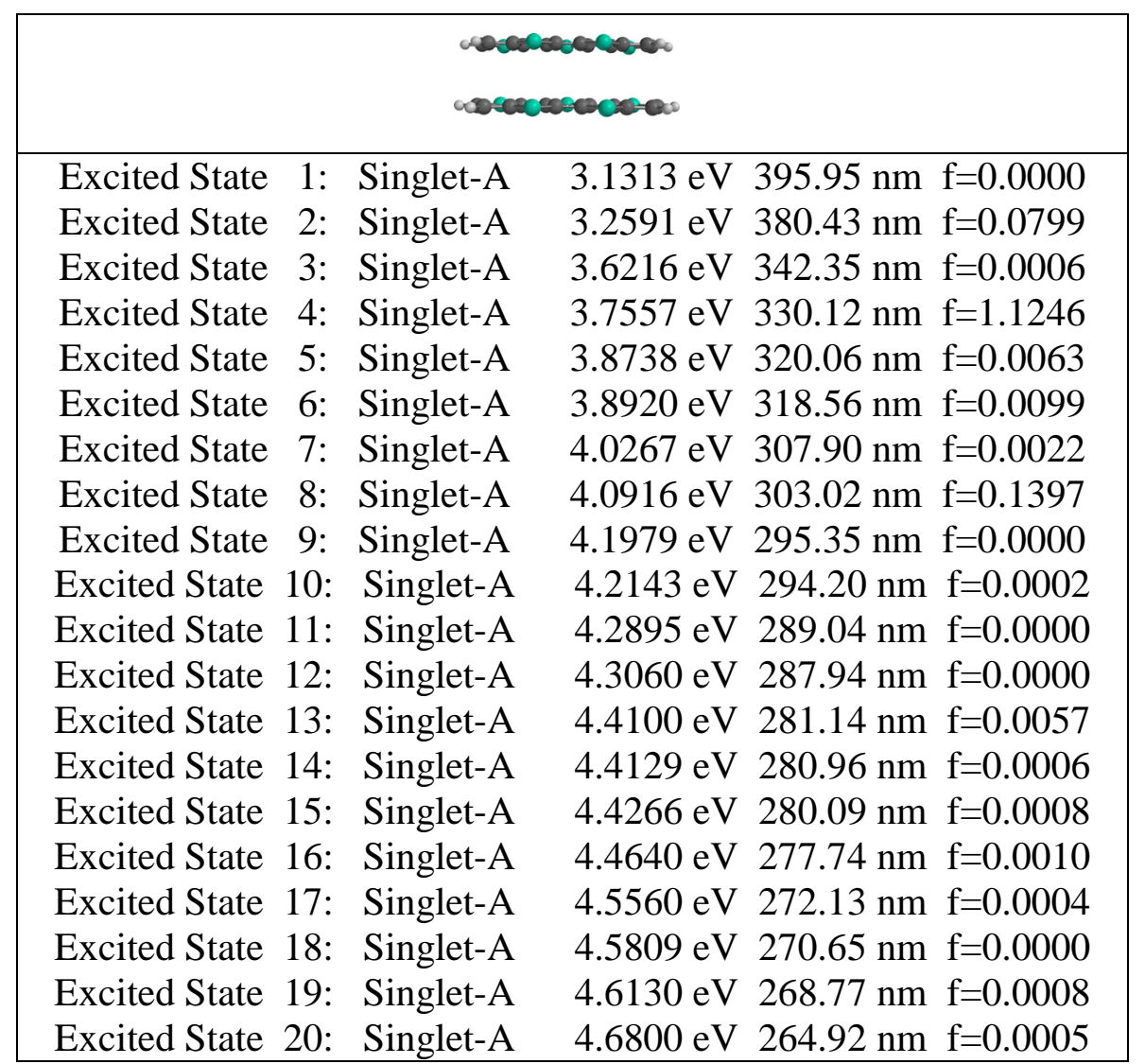

